\title{
Incidencia de un programa sistemático de indoor cycling en la composición corporal de usuarios del gimnasio Smart Fit, de Tunja-Boyacá
}

\section{Incidence of a systematic indoor cycling program on the body composition of users of the gym Smart Fit in Tunja-Boyacá}

César Ferney Bohórquez-Sanabria ${ }^{1}$ (D); María Cristina Bohórquez-González ${ }^{1}$ (D);

Carlos Ramiro Espejo-Erazo ${ }^{1}$ (D)

\begin{abstract}
1Universidad Pedagógica y Tecnológica de Colombia (UPTC). Tunja-Boyacá, Colombia cesarfitbs@gmail.com;
\end{abstract} mbohorquez@jdc.edu.co; Carlos.espejo01@uptc.edu.co

Cómo citar: Bohórquez-Sanabria, C.F.; Bohórquez-González, M.C.; Espejo-Erazo, C.R. 2021. Incidencia de un programa sistemático de indoor cycling en la composición corporal de usuarios del gimnasio smart fit de Tunja-Boyacá. Rev. Digit. Act. Fis. Deport. 7(2):e1829. http://doi. org/10.31910/rdafd.v7.n2.2021.1829

Artículo de acceso abierto publicado por Revista Digital: Actividad Física y Deporte, bajo una licencia Creative Commons CC BY-NC 4.0

Publicación oficial de la Universidad de Ciencias Aplicadas y Ambientales U.D.C.A, Institución de Educación Superior Acreditada de Alta Calidad por el Ministerio de Educación Nacional.

Recibido: febrero 18 de 2021 Aceptado: marzo 21 de 2021 Editado por: Álvaro José Gracia Díaz

\section{RESUMEN}

Introducción: El Indoor Cycling (IC) ha generado un gran desarrollo en los centros de fitness, debido a lo atrayente, dinámico y beneficioso en la calidad de vida de las personas, contribuyendo a disminuir porcentajes elevados de grasa, reclutamiento de importantes grupos musculares, un bajo impacto osteoarticular y un elevado gasto calórico; en tal sentido, se hace necesario llevar a cabo un programa sistemático de Indoor Cycling, que incida satisfactoriamente en la composición corporal de las personas. Objetivo general: Determinar la incidencia de un programa sistemático de Indoor Cycling en la composición corporal de usuarios del gimnasio Smart Fit, de Tunja, Boyacá. Metodología: La presente investigación está dentro del enfoque cuantitativo, con un diseño metodológico cuasi-experimental. Se aplicó un programa sistemático, cuyo objetivo se centra en el diseño de sesiones de Indoor Cycling de 8 semanas, con una frecuencia de 3 sesiones por semana y participaron 30 afiliados al gimnasio Smart Fit, en Tunja, 10 mujeres y 5 hombres del grupo control y la misma cantidad de sujetos del grupo experimental, con una edad promedio de 33,24 \pm
38,47 años, se valoraron a los sujetos objeto de estudio (pre y post) a la aplicación del programa, esto por medio de una báscula de bioimpedancia que permitió registrar la información en una tabla de datos, el \% graso, \% músculo, \% agua, peso e IMC. Resultados: El análisis de los resultados entre los dos grupos con lo que concierne al pre-test y el pos-test, el P-valor de cada una de las variables a contrastar es $>\alpha 0,05$, lo que determina que no alcanza a existir significancia. Conclusión: Según los resultados, el programa de Indoor Cycling no incidió estadísticamente en el análisis del grupo experimental con el grupo control.

Palabras clave: Indoor Cycling; Composición corporal; Actividad física; Estilos de vida y salud.

\section{ABSTRACT}

Introduction: Indoor Cycling (IC) has generated a great development in fitness centres due to the attraction, dynamics and benefits it can have, contributing to reduce high percentages of fat, recruitment of important muscular groups, a low osteoarticular impact and a high caloric expenditure. In this sense, it is necessary to carry out a systematic Indoor Cycling 
programme that satisfactorily affects the body composition of people. Objective: To determine the incidence of a systematic Indoor Cycling programme on the body composition of users of the Smart Fit gymnasium in Tunja-Boyacá. Methodology: This research is within the quantitative approach, with a quasi-experimental design. A systematic 8 week Indoor Cycling programme was developed, with a frequency of 3 sessions per week and 30 members of the Smart Fit gymnasium, in Tunja participated, they were divided into an experimental group and a control group, with an average age of $33.24 \pm 38.47$, evaluations were made (pre and post) this by means of a bioimpedance scale that allowed the recording of $\%$ fat, $\%$ muscle, $\%$ water, weight and I in a data table. M.C. Results: The analysis of the results between the two groups with regard to the pre-test and post-test, the P-value of each of the variables to be contrasted is $>\alpha 0.05$ which determines that there is no significance. Conclusion: According to the results, the Indoor Cycling programme did not statistically affect the analysis of the experimental group with the control group.

Keywords: Indoor Cycling; Body composition; Physical activity; Lifestyles and health.

\section{INTRODUCCIÓN}

La adopción de un hábito en relación con el ejercicio físico es un factor predominante, al punto de prevenir enfermedades crónicas no trasmisibles, derivadas de llevar una vida sedentaria e inactiva físicamente, debido a que, desde el presente estudio, se pretende evitar los riesgos de mortalidad y morbimortalidad; por consiguiente, se diseña e incorpora un programa sistemático de entrenamiento de Indoor Cycling, en el gimnasio Smart Fit, de Tunja - Boyacá (Colombia). Debido a las distintas metodologías de estas sesiones son determinantes en mejorar los indicadores, que constituye la composición corporal (\% grasa, \% músculo, \% agua y otros), como agentes esenciales en manifestar si una persona se encuentra corporalmente saludable o poco saludable.

El interés de este estudio viene dado por el incremento de muertes prematuras, a causa de enfermedades metabólicas, hiperlipidemias, hipertensión arterial, aterosclerosis, infarto de miocardio, entre otras enfermedades crónicas. Según cifras de la Organización Mundial de la Salud
(OMS, 2018), este tipo de enfermedades están repercutiendo, de manera significativa, a países con ingresos bajos y medios; en el mundo, se registran más del 71\% (41 millones) de muertes, a causa de enfermedades no trasmisibles $y$, por lo tanto, es un nuevo desafío, al momento de diseñar un programa sistemático de Indoor Cycling, que cumpla y se ajuste a procedimientos metodológicos, que logren los objetivos planteados.

El Indoor Cycling sería la base que se busca al analizar e incidir significativamente en mantener las recomendaciones, de llevar un porcentaje de grasa saludable. De ahí la importancia de la variabilidad de intensidades en las sesiones, con métodos que permitan combinar un entrenamiento aeróbico y de fuerza, por esa razón, habría mejor obtención en el punto máximo de oxidación de grasas (Fatmax); como lo afirman Randell et al. (2017) es la intensidad a la que se produce la tasa máxima de oxidación de grasas, expresada en valor absoluto (MFO) (g min-1) y en el consumo máximo de oxígeno (VO2 máximo), porque el pilar de las enfermedades crónicas en los seres humanos inicia con tener porcentajes elevados de grasa visceral y subcutánea. Comprender el Indoor Cycling resulta ser un concepto polisémico, entre los más precisos, como lo afirman Barbado \& Barranco (2007), quienes expresan que es una actividad física colectiva, realizada sobre una bicicleta estática al ritmo de la música; se realiza un trabajo predominantemente cardiovascular de alta intensidad, con intervención muy elevada de los grandes grupos musculares del tren inferior. La clase va guiada por un instructor, quien es el responsable de conducir la sesión hacia los objetivos, previamente establecidos. La ejecución de sesiones de Indoor Cycling en usuarios, genera incorporación de diversos parámetros, como la intensidad, la frecuencia, el volumen de entrenamiento y todo lo referente a la periodización, para que se pueda desempeñar la práctica de forma precisa; esto será uno de los pilares predominantes a la hora de obtener cambios en los sujetos del grupo experimental, desde el peso corporal, porcentaje graso, porcentaje masa muscular, porcentaje de agua e índice de masa corporal (IMC). Estos componentes forman parte de la composición corporal que se evaluaron en el transcurso del programa sistemático de Indoor Cycling. De acuerdo con Wilmore \& Costill (2004), la composición corporal es "la estructura química que tiene el cuerpo humano, dentro de esta hay ciertos modelos que lo dividen en sus diversos 
componentes tanto químicos como anatómicos". De esa manera, se puede demostrar los indicadores corporales que posee cada persona, acerca de la constitución de diversos tejidos del cuerpo humano, con el fin de tener un diagnóstico sobre el estado de salud del sujeto.

\section{MATERIALES Y MÉTODOS}

La investigación posee un diseño metodológico cuasiexperimental, con una muestra no probabilística, por conveniencia (Otzen \& Manterola, 2017). El grupo total abordó 30 usuarios, donde 15, hicieron parte del grupo experimental, quienes desarrollaron un programa de Indoor Cycling, mientras los otros 15 usuarios, conformaron el grupo control, que realizaban sesiones de IC, sin ningún tipo de seguimiento; cada uno de los grupos estuvo conformado por 5 sujetos, del sexo femenino y 10 , del masculino, con un rango de edad entre los 20 y los 40 años, además de cumplir con criterio de selección, como el hecho de no presentar lesiones, ni enfermedades que impidieran el cumplimiento del objetivo de estudio, cumplir con el diligenciamiento del cuestionario Par-q and you, tener bicicleta estática o rodillos, tener la disponibilidad y disposición de cumplir semanalmente con 3 sesiones, cada una con una duración de 45 minutos, durante dos mesociclos de tipo entrante y básico desarrollador de 8 semanas, contar con la implementación necesaria para la práctica (Indoor Cycling), facilitar los datos requeridos con fines investigativos, así como la firma del formato de consentimiento informado. Por otra parte, se realizó revisión de literatura científica y de fuentes fiables y con apoyo de está, se definió el tipo, el enfoque, la población, la muestra y los objetivos entre ellos, en pro de generar un nuevo conocimiento, partiendo de hechos teórico - empíricos, para dar respuesta al problema planteado. Finalmente, se obtienen los resultados, de acuerdo con los objetivos trazados, evidenciado en las fases del estudio. Azuero Azuero (2019) afirma que la metodología de la investigación permite descubrir premisas, reconstruir datos a partir de bases teóricas, detallar cada aspecto a desarrollar por el investigador y exponer el tipo de datos, para dar respuesta a los objetivos (Tabla 1.)

Tabla 1. Primera semana programa sistemático de indoor cycling.

\begin{tabular}{|c|c|c|c|c|c|c|}
\hline \multicolumn{7}{|c|}{ TRABAJO I SEMANA (31 agosto - 4 septiembre) } \\
\hline Características & \multicolumn{2}{|c|}{ Día 1} & \multicolumn{2}{|c|}{ Día 2} & \multicolumn{2}{|l|}{ Día 3} \\
\hline Técnica & \multicolumn{2}{|c|}{ Baja intensidad (bi) } & \multicolumn{2}{|c|}{ Intervalo extensivo (ie) } & \multicolumn{2}{|l|}{ Fartlek (ft) } \\
\hline Intensidad & \multicolumn{2}{|c|}{$65 \%-75 \%$} & \multicolumn{2}{|c|}{$65 \%-85 \%$} & \multicolumn{2}{|l|}{$65 \%-85 \%$} \\
\hline Intensidad/rpm & \multicolumn{2}{|l|}{ 60-80rpm } & \multicolumn{2}{|l|}{ 80-100rpm } & \multicolumn{2}{|l|}{ 80-100rpm } \\
\hline Duración & \multicolumn{2}{|l|}{45 minutos } & \multicolumn{2}{|l|}{45 minutos } & \multicolumn{2}{|l|}{45 minutos } \\
\hline Fase inicial & $\begin{array}{l}\text { Activación } \\
\text { muscular }\end{array}$ & $\begin{array}{l}15 \\
\min \end{array}$ & $\begin{array}{l}\text { Activación } \\
\text { muscular }\end{array}$ & $\begin{array}{l}15 \\
\min \end{array}$ & $\begin{array}{l}\text { Activación } \\
\text { muscular }\end{array}$ & $\begin{array}{l}15 \\
\min \end{array}$ \\
\hline Fase central & $\begin{array}{l}\text { Trabajo } \\
\text { específico (bi) }\end{array}$ & $\begin{array}{l}25 \\
\min \end{array}$ & $\begin{array}{l}\text { Trabajo específico } \\
\text { (ie) }\end{array}$ & $\begin{array}{l}25 \\
\min \end{array}$ & $\begin{array}{l}\text { Trabajo } \\
\text { específico (ft) }\end{array}$ & $\begin{array}{l}25 \\
\min \end{array}$ \\
\hline Fase final & Estiramientos & $\begin{array}{l}10 \\
\min \end{array}$ & Estiramientos & $\begin{array}{l}10 \\
\min \end{array}$ & Estiramientos & $\begin{array}{l}10 \\
\min \end{array}$ \\
\hline
\end{tabular}

Nota. Planificación de la primera semana del programa sistemático de Indoor Cycling, del 31 de agosto al de septiembre de 2020.

\section{RESULTADOS Y DISCUSIÓN}

Posteriormente, se lleva a cabo un estudio de tipo descriptivo, para encontrar aspectos propios de la población, tales como la homogeneidad, la heterogeneidad, la desviación estándar y la media, como valores esenciales de preferencia central. Seguidamente, se efectuó la prueba de ShapiroWilk, para determinar normalidad en las pruebas, teniendo en cuenta, que la muestra del estudio es de un total de 30 personas, en edades entre 20 a 40 años. Posteriormente, se llevó a cabo la estadística inferencial que, para el caso fue la prueba paramétrica, denominada T de Student; para muestras relacionadas y para pruebas no paramétricas, se utilizó Wilcoxon. Dicho estudio, se ejecutó de acuerdo con los datos obtenidos, los que se recolectaron de manera manual y se sistematizaron en Microsoft Excel Posteriormente, se exportaron a SPSS v.21.0, para su procedimiento estadístico y posterior análisis. 
Análisis estadístico de grupo control y grupo intervención. En esta sección, se llevó a cabo un análisis descriptivo de cada una de las variables, teniendo en cuenta las medidas de preferencia central (media, varianza y desviación estándar).

En la tabla 2, se puede observar, en el género femenino, que la masa grasa, el agua corporal y la grasa visceral es menor en el grupo intervención que en el grupo control, mientras que la masa libre de grasa, la masa muscular es mayor en las mujeres del grupo control y la masa ósea es igual en ambos grupos. En cuanto al género masculino, la figura detalla cómo la masa libre de grasa y el porcentaje de masa muscular es mayor en el grupo intervención; por otro lado, el agua corporal y la grasa visceral es mayor en el grupo control; casualmente, la masa grasa y la masa ósea en los hombres de ambos grupos, es igual.

Tabla 2. Análisis estadístico de los indicadores de la composición corporal del grupo control y grupo intervención.

\begin{tabular}{|c|c|c|c|c|c|c|c|}
\hline \multicolumn{2}{|c|}{ Intergéneros } & \multirow{2}{*}{$\begin{array}{c}\begin{array}{c}\text { Masa, } \\
\text { Kg }\end{array} \\
10 \\
\end{array}$} & \multirow{2}{*}{$\begin{array}{c}\text { Masa } \\
\text { grasa } \\
\%\end{array}$} & \multirow{2}{*}{$\begin{array}{c}\begin{array}{c}\text { Grasa } \\
\text { visceral } \\
\%\end{array} \\
10 \\
\end{array}$} & \multirow{2}{*}{$\begin{array}{c}\text { Masa libre } \\
\text { de grasa }\end{array}$} & \multirow{2}{*}{$\begin{array}{c}\begin{array}{c}\text { Masa } \\
\text { muscular \% }\end{array} \\
10 \\
\end{array}$} & \multirow{2}{*}{$\begin{array}{c}\begin{array}{c}\text { Agua } \\
\text { corporal \% }\end{array} \\
10 \\
\end{array}$} \\
\hline \multirow{4}{*}{ 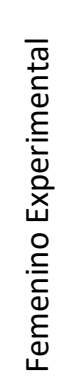 } & $\mathrm{N}$ & & & & & & \\
\hline & Media & 2,070 & 28,070 & 2.500 & 39,970 & 37,700 & 50,600 \\
\hline & Varianza & 0,018 & 38,905 & 1.386 & 7,976 & 7,309 & 44,796 \\
\hline & $\begin{array}{c}\text { Desviación } \\
\text { estándar }\end{array}$ & 0,1337 & 6,2374 & 1.1785 & 2,8241 & 2,7035 & 6,6929 \\
\hline \multirow{4}{*}{ 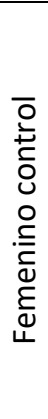 } & $\mathrm{N}$ & 10 & 10 & 10 & 10 & 10 & 10 \\
\hline & Media & 2,100 & 29,100 & 3.300 & 39,620 & 36,020 & 52,970 \\
\hline & Varianza & 0,009 & 71,222 & 3.567 & 4,728 & 7,042 & 23,838 \\
\hline & $\begin{array}{c}\text { Desviación } \\
\text { Estándar }\end{array}$ & 0,0943 & 8,4393 & 1.8886 & 2,1745 & 2,6536 & 4,8824 \\
\hline \multirow{4}{*}{ 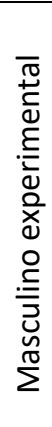 } & $\mathrm{N}$ & 5 & 5 & 5 & 5 & 5 & 5 \\
\hline & Media & 3,000 & 27,340 & 3.800 & 58,220 & 44,640 & 51,940 \\
\hline & Varianza & 0,018 & 38,905 & 1.200 & 1,897 & 92,283 & 79,533 \\
\hline & $\begin{array}{c}\text { Desviación } \\
\text { Estándar }\end{array}$ & 0,1414 & 9,3136 & 1.0954 & 1,3773 & 9,6064 & 8,9181 \\
\hline \multirow{4}{*}{ 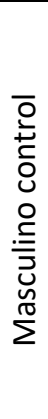 } & $\mathrm{N}$ & 5 & 5 & 5 & 5 & 5 & 5 \\
\hline & Media & 3,040 & 27,340 & 4.200 & 56,880 & 38,800 & 52,880 \\
\hline & Varianza & 0,033 & 86,743 & 1.700 & 1,897 & 32,735 & 7,222 \\
\hline & $\begin{array}{c}\text { Desviación } \\
\text { Estándar }\end{array}$ & 0,1817 & 8,3388 & 1.3038 & 1,3773 & 5,7215 & 2,6874 \\
\hline
\end{tabular}

Nota. Análisis estadístico del grupo experimental y grupo control femenino/masculino. 


\section{Revista Digital: Actividad Física y Deporte}

Caracterización del grupo. En la caracterización del grupo, se observa que existe una concentración de casi el $50 \%$ de individuos, en el rango de edad de los 33 a los 38 años; la edad promedio del grupo intervención es de 32 años, mientras que la edad promedio del grupo control, es de 30 años.

Resultado pretest y postest. La prueba de normalidad de Shapiro Wilk permite analizar la distribución de los datos cuando la muestra es menor a 50 datos, información que es requerida para determinar la estadística paramétrica o no paramétrica que corresponda.

Como se observa en la tabla 2, las variables pretest y pos-test de grasa y músculo son menores al Pvalor 0,05 , mientras que las variables pre-test y pos-test del porcentaje de agua, peso e IMC son mayores que $\mathrm{P}$-valor 0,05.
Además, se exponen los datos de las variables pre-test y pos-test de grasa y músculo no tienen una distribución normal, por tal razón, se determina que se debe utilizar la prueba estadística no paramétrica Wicolxon, para comparar dos medidas de los mismos sujetos del mismo grupo; es recomendable con irmar la distribución de los datos, con una variable de diferencia de signos, porque Wilcoxon evalúa la significancia entre la sumatoria de rangos.

Los datos de las variables pre-test y pos-test de grasa y músculo tienen una distribución normal, lo que indica que es necesario hacer el análisis estadístico inferencia con la prueba paramétrica $t$ de Student, para muestras relacionadas (Tabla 3; Figura 1).

Tabla 3. Prueba de Normalidad de Shapiro Wilk.

\begin{tabular}{|l|c|c|c|}
\hline \multirow{2}{*}{} & \multicolumn{3}{|c|}{ SHAPIRO-WILK } \\
\cline { 2 - 4 } & Estadístico & gl. & Significancia \\
\hline Pre-test grasa & 0,801 & 15 & 0,004 \\
\hline Pre-test músculo & 0,706 & 15 & 0,000 \\
\hline Pre-test agua & 0,920 & 15 & 0,192 \\
\hline Pre-test peso & 0,944 & 15 & 0,437 \\
\hline Pre-test IMC & 0,933 & 15 & 0,305 \\
\hline Post-test grasa & 0,867 & 15 & 0,031 \\
\hline Post-test músculo & 0,737 & 15 & 0,001 \\
\hline Post-test agua & 0,939 & 15 & 0,371 \\
\hline Post-test peso & 0,937 & 15 & 0,345 \\
\hline Post-test IMC & 0,898 & 15 & 0,089 \\
\hline
\end{tabular}

Nota. La tabla muestra el límite inferior de la significancia verdadera. A. Corrección de significancia propuesta por Lilliefors.

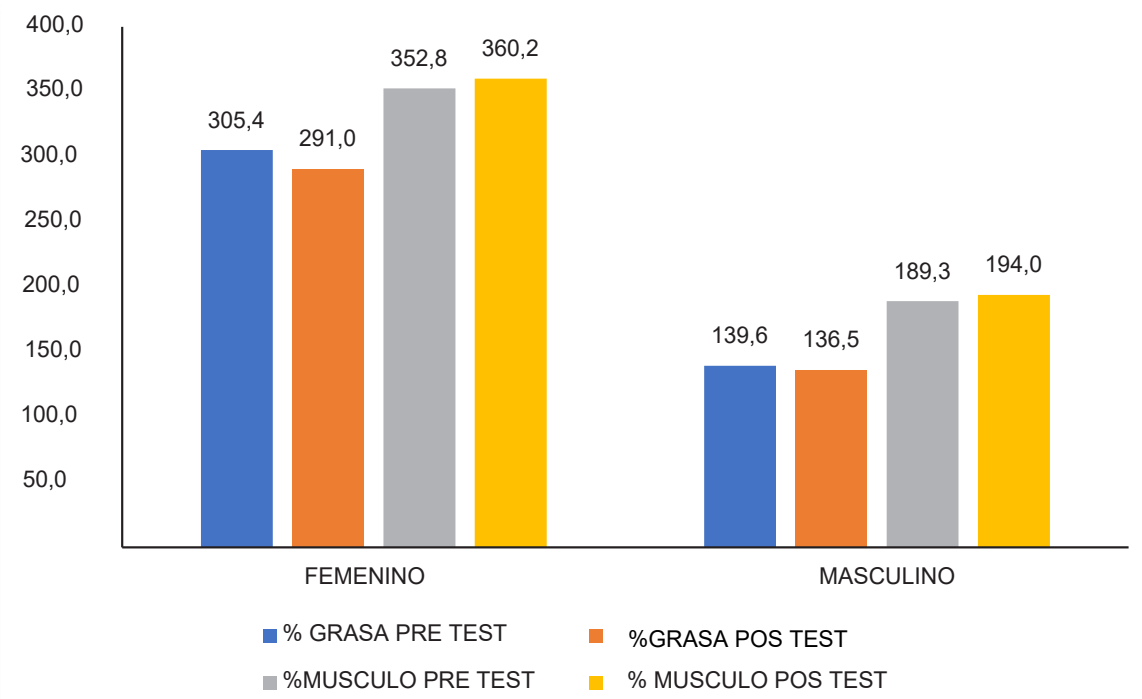

Figura 1. Comparación entre el \% de grasa por géneros y comparación del \% de masa muscular por géneros.

Nota. Relación entre los indicadores de la composición corporal como un \% Grasa y el \% Músculo. 
Uno de los indicadores más fundamentales en la composición corporal y, del cual, permitió generarse un cambio significativo, es en la masa grasa, ya que, al hacer el análisis individual con el grupo experimental, resulta ser muy significativo el programa, esto, al tener un $p=<0,05$. El tejido muscular se vio desarrollado, debido a la variedad de metodologías que se utilizaban en las sesiones de Indoor Cycling, por medio de técnicas propias del IC, como fartlek, challenge, montaña, intervalos extensivos, intervalos intensivos y otros (Figura 2).

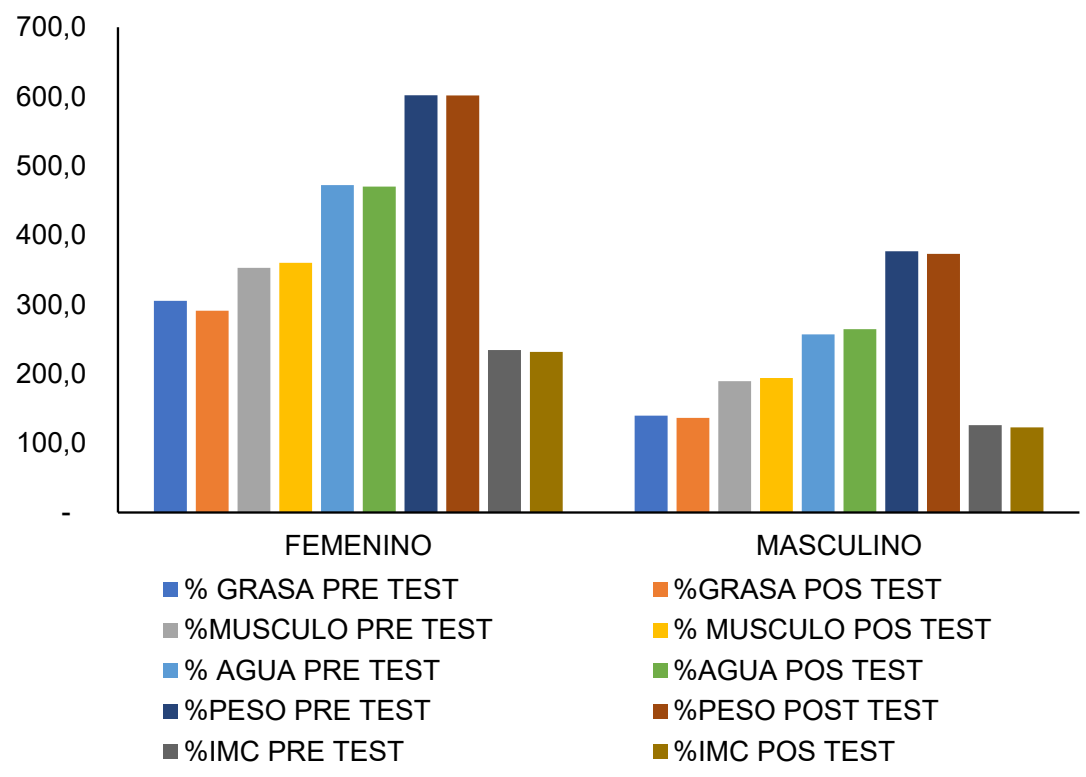

Figura 2. Incidencia en la composición corporal pre y post del Programa Sistemático de Indoor Cycling, en el grupo experimental.

Nota. Datos pre y post de los indicadores de la composición corporal del grupo experimental.

Se pudo llevar a cabo la progresión significativa desde el inicio del programa, una segunda toma al intermedio y una última toma de datos, al finalizar el programa. El análisis individual del grupo de intervención requiere de un proceso metodológico, claramente estructurado, que busca, a través de pruebas paramétricas y no paramétricas, demostrar si otros programas o métodos de entrenamiento pueden lograr también incidir sobre la composición corporal; las gráficas de barra comprueban la direccionalidad o neutralidad de la significancia de la inferencia, de cada una de las pruebas estadísticas.

En la tabla 4 del grupo control, se evidencia un límite inferior de la significación verdadera, que permite evidenciar los datos previos, durante y posterior a la investigación, debido a que el grupo control no siguió el direccionamiento y la secuencialidad del programa sistemático de Indoor Cycling, por lo tanto, ellos destinaban sus sesiones empíricamente y por medio de videos.

Tabla 4. Prueba de normalidad por género, según el grupo control.

\begin{tabular}{|l|l|c|c|c|}
\hline \multirow{2}{*}{ Género } & \multicolumn{3}{|c|}{ Shapiro-Wilk } \\
\cline { 3 - 5 } & & Estadístico & Gl & Sig. \\
\hline \multirow{2}{*}{ Pre-test grasa } & Femenino & 0,928 & 10 & 0,431 \\
\cline { 2 - 5 } & Masculino & 0,960 & 5 & 0,808 \\
\hline \multirow{2}{*}{ Pre-test agúsculo } & Femenino & 0,808 & 10 & 0,160 \\
\cline { 2 - 5 } & Masculino & 0,856 & 5 & 0,213 \\
\hline \multirow{2}{*}{ Pre-test peso } & Femenino & 0,949 & 10 & 0,655 \\
\cline { 2 - 5 } & Masculino & 0,923 & 5 & 0,551 \\
\hline \multirow{2}{*}{ Pre-test IMC } & Femenino & 0,932 & 10 & 0,470 \\
\cline { 2 - 5 } & Masculino & 0,906 & 5 & 0,444 \\
\hline \multirow{2}{*}{ Pre-test grasa } & Femenino & 0,917 & 10 & 0,330 \\
\cline { 2 - 5 } & Masculino & 0,959 & 5 & 0,802 \\
\cline { 2 - 5 } & Memenino & 0,892 & 10 & 0,178 \\
\hline
\end{tabular}

Nota. Límite inferior de la significancia verdadera de algunos indicadores de la composición corporal. 


\section{Revista Digital: Actividad Física y Deporte}

Como se observa en la tabla 5 , todas las variables tienen un P-valor, que superan el de $\alpha=0,05$, tanto para todos los sujetos como para los sujetos según género. Ya que la distribución de los datos es normal, se escoge la prueba paramétrica t-Student, para muestras relacionadas, con el fin de comparar las mediciones antes y después de 8 semanas sin la intervención del programa sistemático, solamente el direccionamiento de sesiones de Indoor Cycling virtuales.

Tabla 5. Prueba de normalidad de la totalidad de individuos del grupo control.

\begin{tabular}{|l|c|c|c|}
\hline \multirow{2}{*}{\multicolumn{1}{|c|}{ Género }} & \multicolumn{3}{|c|}{ Shapiro-Wilk } \\
\cline { 2 - 4 } & Estadístico & Gl & Sig. \\
\hline Pre-test grasa & 0,932 & 15 & 0,295 \\
\hline Pre-test músculo & 0,912 & 15 & 0,146 \\
\hline Pre-test agua & 0,956 & 15 & 0,622 \\
\hline Pre-test peso & 0,965 & 15 & 0,772 \\
\hline Pre-test IMC & 0,908 & 15 & 0,126 \\
\hline Post-test grasa & 0,912 & 15 & 0,145 \\
\hline Post-test músculo & 0,909 & 15 & 0,132 \\
\hline Post- test agua & 0,978 & 15 & 0,957 \\
\hline Post-test peso & 0,969 & 15 & 0,837 \\
\hline Post-test IMC & 0,937 & 15 & 0,346 \\
\hline
\end{tabular}

Nota. Prueba Normalidad de las variables del grupo control.

La figura 3 presenta que la progresión corporal sesiones que estén debidamente planificadas durante la toma de datos antes, durante y después y estructuradas, permitirán en el sujeto mejores de 8 semanas, tiempo en que duró la investigación efectos en la composición corporal. fue muy insignificante $>0,5$, lo que demuestra que,

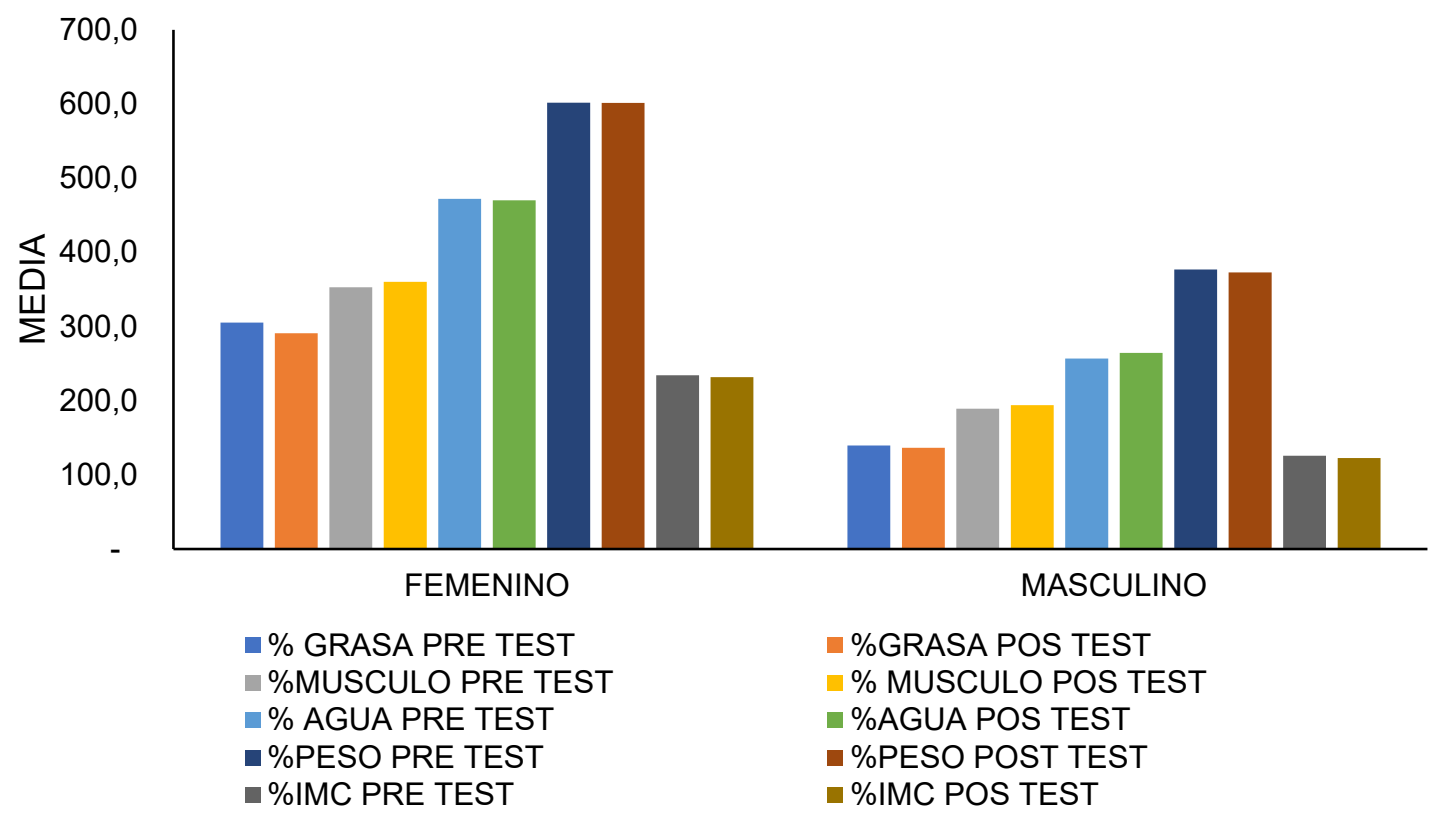

Figura 3. Comparación total entre las variables de estudio de la composición corporal según genero del grupo control.

Nota. Datos pre y post de los indicadores de la composición corporal del grupo cont 
Análisis comparativo entre el grupo control y el grupo experimental. El análisis comparativo entre grupos requiere de un proceso metodológico claramente estructurado, que busca, a través de pruebas paramétricas y no paramétricas, demostrar si otros programas o métodos de entrenamiento pueden lograr también incidir sobre la composición corporal, tales como intervalos intensivos, intervalos extensivos, fartlek, entre otras técnicas, propias del Indoor Cycling; las gráficas de barra comprueban la direccionalidad o neutralidad de la significancia de la inferencia, de cada una de las pruebas estadísticas (Tabla 6).

Tabla 6. Prueba de normalidad entre grupo experimental y grupo control.

\begin{tabular}{|c|l|c|c|c|}
\hline \multirow{2}{*}{ GRUPO } & \multicolumn{3}{|c|}{ Shapiro-Wilk } \\
\cline { 3 - 5 } & & Estadístico & GI & Sig. \\
\hline \multirow{2}{*}{$\%$ Grasa } & Experimental & 0,867 & 15 & 0,031 \\
\cline { 2 - 5 } & Control & 0,912 & 15 & 0,145 \\
\hline \multirow{2}{*}{$\%$ Músculo } & Experimental & 0,737 & 15 & 0,001 \\
\cline { 2 - 5 } & Control & 0,909 & 15 & 0,132 \\
\hline \multirow{2}{*}{$\%$ Agua } & Experimental & 0,939 & 15 & 0,371 \\
\cline { 2 - 5 } & Control & 0,308 & 15 & 0,000 \\
\hline \multirow{2}{*}{ Peso } & Experimental & 0,339 & 15 & 0,000 \\
\cline { 2 - 5 } & Control & 0,969 & 15 & 0,837 \\
\hline \multirow{2}{*}{ IMC } & Experimental & 0,898 & 15 & 0,089 \\
\cline { 2 - 5 } & Control & 0,937 & 15 & 0,346 \\
\hline
\end{tabular}

Nota. Indicadores de la composición en los dos grupos de la investigación, grupo experimental y grupo control.

Se analiza el P-valor de ambos grupos por cada variable y se espera que los dos sean mayores que $\alpha=0,05$ para determinar si los datos se distribuyen de manera homogénea; los resultados de la prueba de Shapiro Wilk demuestran, según la significancia bilateral asintótica, que el P-valor no concuerda entre los grupos, en ninguna de las variables a estudiar de la composición corporal, entre los grupos. Dado que no concuerdan el P-valor en ninguna variable entre el grupo de intervención y el grupo control, estadísticamente, no hay normalidad en la distribución de los datos, por lo tanto, se recomienda utilizar una prueba no paramétrica que, para este caso, corresponderá a la U de Mann Whitney.

En la figura 4, se comparan los resultados de grasa, músculo y agua, entre el grupo experimental y grupo control femenino; en el grupo experimental femenino presenta resultados más representativos, en cuanto a los indicadores de la composición corporal, como porcentaje (\%) grasa, músculo y agua, pero, estadísticamente, no alcanzan a ser significativos, debido a factores endógenos y exógenos, durante la investigación (Figura 5).

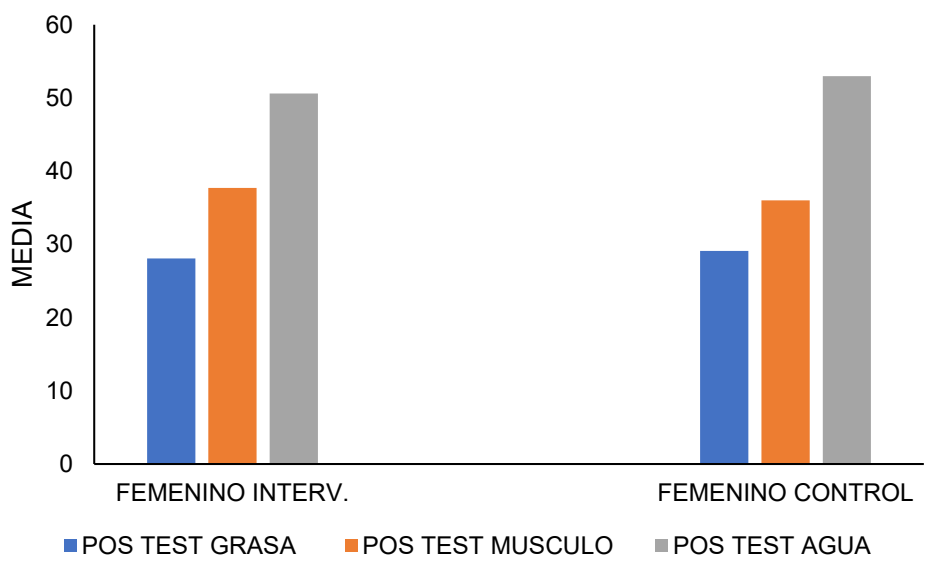

Figura 4. Comparación de variables de la composición corporal entre mujeres del grupo experimental con el grupo control.

Nota. Relación de la grasa, músculo y agua en la composición corporal del grupo intervención y control de mujeres. 


\section{Revista Digital: Actividad Física y Deporte}

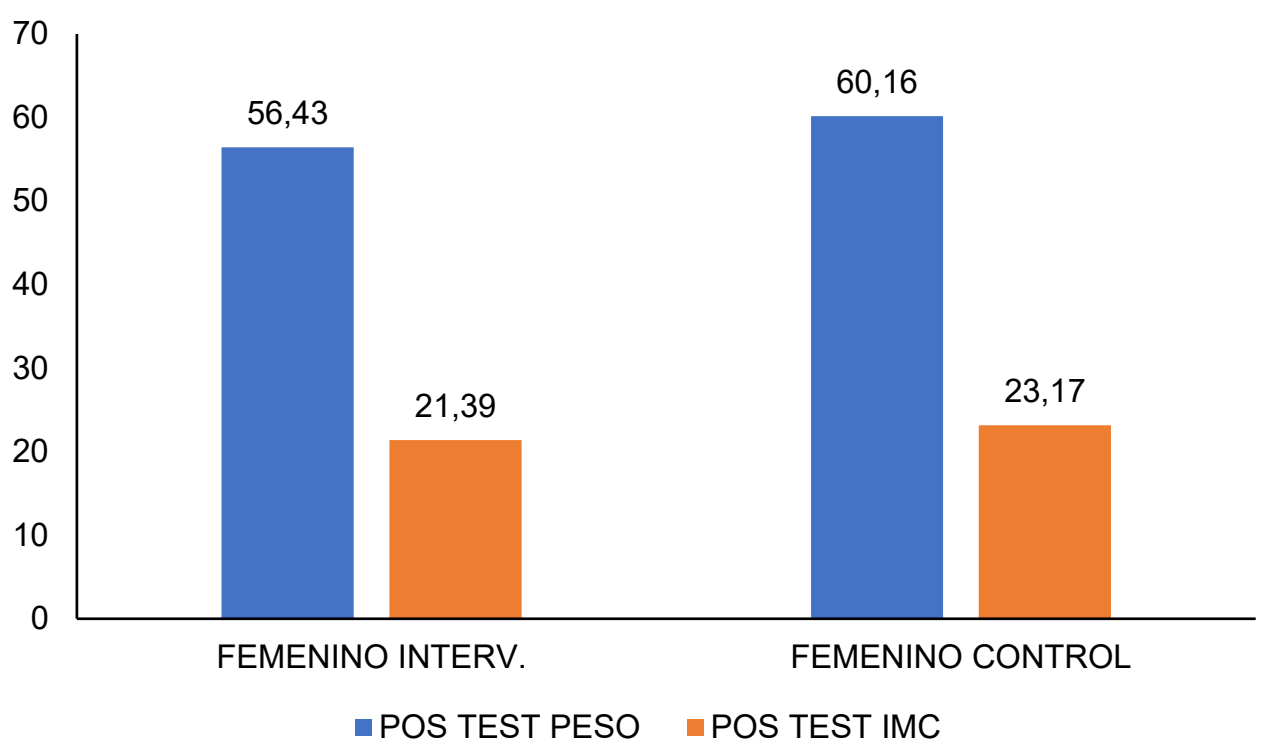

Figura 5. Comparación de variables del peso e IMC entre mujeres del grupo experimental con el grupo control.

Nota. Relación del peso en la composición corporal del grupo intervención y control de mujeres.

Comparación de las variables que hacen parte de la composición corporal, entre el género masculino del grupo intervención, con el grupo control: prueba de Mann-Whitney, para comparación de variables de la composición corporal del género masculino, entre los dos grupos de estudio.

En la figura 6, se comparan los resultados de grasa, músculo y agua, entre el grupo experimental y el grupo control masculino, en el que el grupo experimental masculino presenta mejores resultados, en cuanto al porcentaje (\%) grasa, músculo y agua, pero, estadísticamente, no son significativos, debido al tiempo de ejecución, a mayor tiempo, existiría una significancia más prevalente.

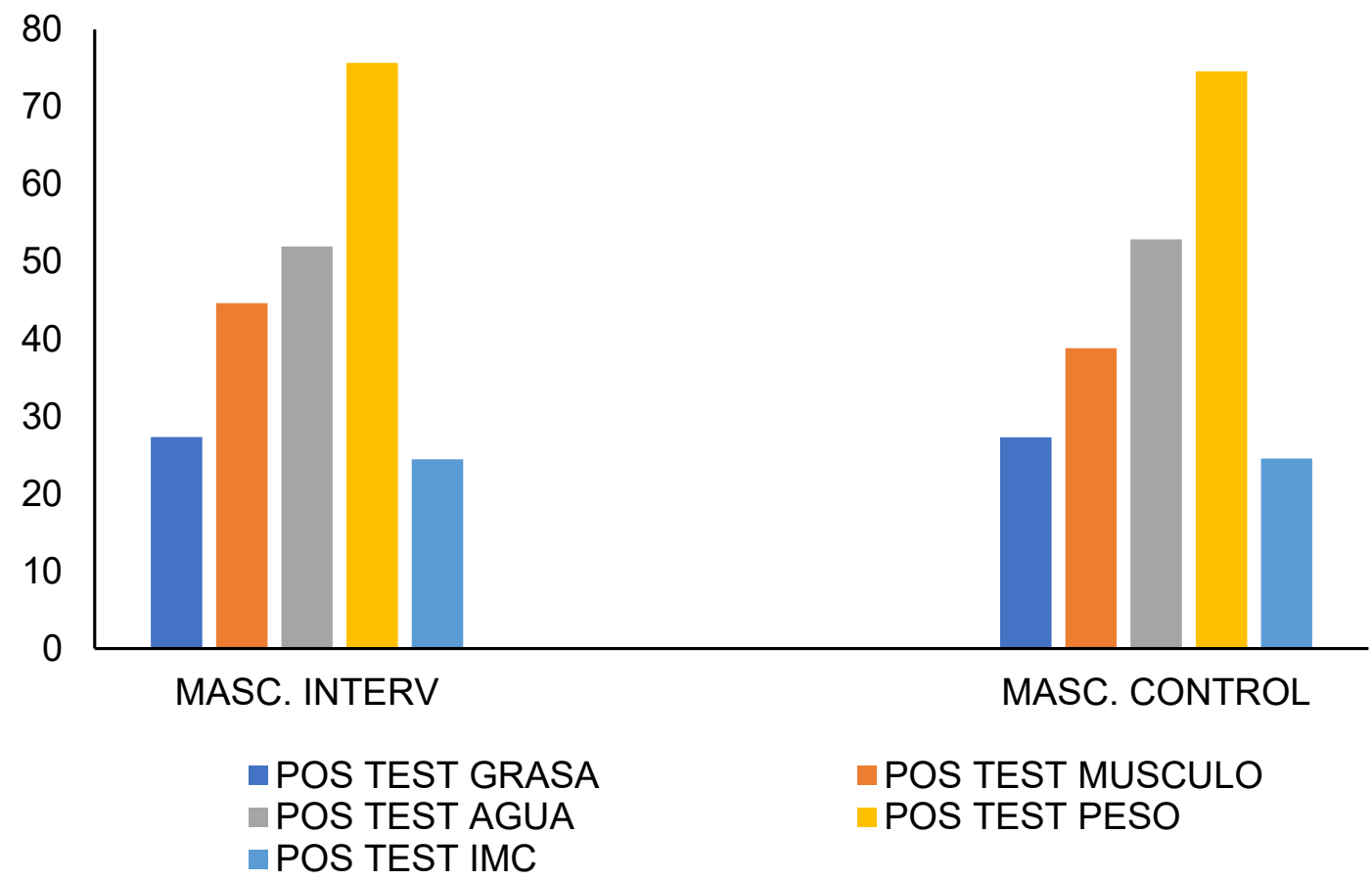

Figura 6. Comparación de variables del peso e IMC entre hombres de los grupos de estudio. Nota. Relación de los indicadores de la com posición corporal del grupo intervención y control de hombres. 
A partir de este estudio, se pudieron determinar cambios que, estadísticamente son significativos $(p \leq, 05)$ y esto se dio, mediante el análisis individual del grupo experimental con lo que respecta a la intervención en el diseño del programa sistemático de Indoor Cycling, en relación con la composición corporal; sin embargo, el objetivo base de esta investigación, en el análisis del grupo de intervención y grupo control, los resultados no son estadísticamente significativos en la composición corporal $(p \geq 05)$ de los sujetos del grupo intervención, en relación con el grupo control, posterior a la implementación del programa sistemático de Indoor Cycling, valorado por medio de la báscula Tanita BC-1500 Ironman e incorporando características propias de la planificación desde frecuencia, intensidad, duración, técnica y más. Con relación a la composición corporal, los lineamientos concuerdan con lo proyectado, según Vilarinho et al. (2009), en el que pretendían, por medio del Indoor Cycling, obtener un efecto en la composición corporal, la resistencia muscular, el balance y actividades diarias, en personas mayores y físicamente activas, donde se efectuó una intervención de 12 semanas, basada en valoraciones pre y post de la investigación. Con lo que respecta a la composición corporal, no presentaron mejoras significativas $(p \geq 0,05)$, los resultados fueron provechosos, pero no lo suficiente para que, en la parte estadística, abordara ello; a mayor tiempo de aplicación, la investigación hubiese tenido una mejor trascendencia, en razón de que el Indoor Cycling es una de las actividades que presenta cuantiosas ventajas extrínsecas, como fortalecimiento del tejido muscular, articular y óseo, mejora del sistema cardiovascular, disminución del porcentaje graso, mejora el sistema inmunológico, entre otros e intrínsecas, tales como reducción del estrés, liberación de serotonina y dopamina, hormonas encargadas de generar un estado anímico, más vital en el ser humano.

En la intervención del programa sistemático de Indoor Cycling, en la que se mantenía una frecuencia de 3 veces por semana, cada sesión constaba de 45-60 minutos, se pretendía ir incrementando y variando las intensidades, cargas y metodologías durante las 8 semanas, que estaba destinada dicha actividad, con el fin de obtener cambios beneficiosos en la composición corporal. Los resultados estadísticos evidencian que, haciendo un análisis exclusivamente del grupo de intervención, son representativos ( $p \leq, 05)$; asimismo, lo menciona Kaya et al. (2018), al realizar una intervención del entrenamiento de Indoor
Cycling de 6 semanas de duración, en la composición corporal de las mujeres; a partir de la tercera semana, se empezaron a evidenciar cambios significativos. En cuanto al grupo de esta investigación, evidenciaba mejoras en el peso corporal $(p<0,01)$, el porcentaje de agua y el porcentaje graso tuvo significancia desde la cuarta semana, del $p<0,01$; respecto a la investigación base, el peso corporal tuvo significancia del $p<0,4$, al igual que el porcentaje de agua, que abordó un mejoramiento del $p<0,19$ y la masa grasa fue representativo, en un $p<0,004$. Luego de las 6 semanas, se observaron cambios positivos en los diversos componentes, que constituye la composición corporal.

El Indoor Cycling, al ser una modalidad del Fitness, presentó multiplicidad de características positivas, de las cuales, permitieron brindar compensación en las condiciones que integra la composición corporal; dentro de este orden de ideas, Chavarrias et al. (2019) ratifican los efectos de clases de fitness de 8 semanas sobre la composición corporal y la aptitud física. Tuvo como propósito comparar los efectos de 8 semanas de Indoor Cycling en la composición corporal, en la que experimentaron resultados significativos, desde una disminución en el peso corporal (BM) y el porcentaje de masa grasa corporal (BFM) $(p<0,004)$, en comparación con el programa sistemático de Indoor Cycling. En la composición corporal muestra que el grupo de intervención tuvo una reducción del porcentaje de grasa, en un $p<0,008$; en cuanto al peso corporal, también se evidenciaron cambios significativos $(p<0,43)$, lo que afirma que la incorporación de una modalidad de fitness (Indoor Cycling), demuestra gran incidencia sobre la composición corporal; no obstante, en la comparación con el grupo control, no resultó estadísticamente significativo.

Algunos factores externos impactan en el programa, representativamente, para que los resultados sean aún más eficientes; la respuesta de la aplicación del programa sistemático de Indoor Cycling, en la composición corporal, durante 8 semanas, no fue significativo $(p>, 05)$, debido a que, para que existiera significancia, se requiere de mayor tiempo y, a su vez, de componentes que inciden endógenamente, en la persona que realiza esta actividad. En comparación con el estudio de Valle et al. (2010), existió un impacto al indagar los efectos de la dieta y el Indoor Cycling, en la composición corporal, que buscaba analizar la composición corporal en personas después de doce semanas de entrenamiento de Indoor Cycling y, de los 
cuales, se obtuvo que los grupos experimentales de Indoor Cycling $(\mathrm{Cl})$ e Indoor Cycling, asociado a dieta hipocalórica $(C D)$, mejoraron, significativamente, los efectos en la composición corporal, después de 12 semanas de intervención (masa corporal, porcentaje de grasa e índice de masa corporal) $(p<0,05)$. a $(p=0,02)$, tuvo un desarrollo relevante para los grupos de Indoor Cycling, con la implementación que agentes externos incidieran, en gran medida, en los componentes de la composición corporal, especialmente, en el porcentaje graso de los usuarios, porque a mayor cantidad de grasa visceral y subcutánea que tenga una persona, más probabilidad hay de desencadenar enfermedades no transmisibles; mantener un equilibrado porcentaje de grasa es equivalente a una persona saludable.

Para que existan cambios positivos en la composición corporal de los sujetos de intervención, se condicionaron mediante los principios de entrenamiento, porque representan una función muy indispensable, en el momento del diseño del programa sistemático de Indoor Cycling, debido a que de esto depende, si la planificación del entrenamiento fue la adecuada y si se incluyeron las características necesarias, a su vez, si existió un diagnóstico eficaz a tener en cuenta y que incidió en el principio de individualización, donde se requería conocer el efecto biológico, que es capaz de resistir el sujeto; asimismo, el principio de continuidad en que los usuarios destinados a la investigación debieron ser constantes, para obtener resultados óptimos. Del mismo modo, el principio de progresión, se incorpora como mecanismo para incrementar la carga de la sesión, que estimula el umbral del sujeto, para que vuelva a producir reacciones en el organismo; son uno de los principios que se implementaron en la investigación, para generar efectos en la composición corporal $(p<0,05)$ de las personas que realizaron el programa, como lo afirma Abraldes Valeiras (2016). Los principios biológicos son los que influyen en el proceso de adaptación del organismo de la persona que ejecuta la actividad; en otras palabras, esos efectos generan una adaptación endógena, que experimentan los organismos, cuando son sometidos a cargas en la sesión o entrenamiento.

\section{CONCLUSIONES}

Para los sujetos objeto de estudio que, en este caso, hacían parte del grupo experimental, la incidencia del programa sistemático de Indoor Cycling de 8 semanas, muestra que la significancia es $<0,5$, es decir, que se obtuvieron mejorías en la composición corporal, al realizar, únicamente, el análisis del grupo experimental, del sexo femenino y masculino.

Al hacer ese mismo análisis estadístico, pero con los dos grupos de la investigación, los sujetos del grupo experimental con los sujetos del grupo control, la incidencia del programa sistemático de Indoor Cycling fue $>0,5$, lo cual, indica que, estadísticamente, no existieron diferencias significativas entre los dos grupos, porque los integrantes del grupo control obtuvieron ciertos cambios, pero en menor magnitud y al no ser más largo el programa, no pudo evidenciar significativamente el cambio de los dos grupos.

En el análisis de los resultados de los indicadores del porcentaje de masa grasa, el peso corporal, el índice de masa corporal y el porcentaje de agua, al obtener una disminución, reflejaron los cambios favorables en la salud de los sujetos del grupo experimental y, de la misma forma, se efectuó un mejoramiento de la masa magra de los participantes de este grupo.

La práctica del IC realizado en forma sistemática y direccionada es una estrategia que permite controlar el estado de los indicadores de la composición corporal; de igual manera, se pueden prevenir enfermedades crónicas no transmisibles, que conlleva a alteraciones de masa grasa en la estructura corporal de las personas y, así, afianzar hábitos de vida saludables e impulsar personas sanas y vitales, en la vida de cada uno de ellos.

El tiempo de la investigación incidió considerablemente, debido a que la duración fue condicionada en 8 semanas, tiempo que, científicamente, se ha determinado que se originan los primeros cambios estructurales en los sujetos del grupo experimental.

En lo que respecta a la práctica de Indoor Cycling, a mayor tiempo de práctica direccionada y contemplando los principios del entrenamiento, mayor significancia en la ganancia de los beneficios, que competen a los componentes de la composición corporal.

En el análisis del grupo de experimental y grupo control, se evidenció que el grupo que efectuó el programa sistemático de Indoor Cycling, que pudo tener mayor significancia en los resultados, si el programa hubiera tenido un tiempo de intervención 
más extenso, pero aun así, los sujetos quienes realizaban sesiones de Indoor Cycling mantenían un orden continuo y progresivo, en busca de afianzar y sustentar las bases científicas y las teorías que han intervenido en estudios, en lo que se tiene, como finalidad, mejorar, por medio de una actividad o ejercicio físico, las condiciones extrínsecas de las personas.

Al realizar el análisis comparativo entre las mujeres del grupo experimental y del grupo control, los resultados evidencian que, en las primeras, disminuyó el porcentaje de la grasa, obtuvieron mayor proporción de masa muscular, menor porcentaje de agua, menor peso y menor IMC, pese a que, estadísticamente, la significancia no es representativa. En el caso del grupo experimental y control de sexo masculino, de acuerdo con los resultados, se puede afirmar claramente que, el primer grupo, es decir, quienes desarrollaron el programa, presentaron en la última prueba, disminución de la masa grasa, mayor porcentaje de masa muscular, menor porcentaje de agua, reducción de peso y menor IMC.

Incorporar factores desencadenantes en la mejora de indicadores de la composición corporal, brinda a las próximas investigaciones, la capacidad de intervenir, acerca de la caracterización del ejercicio físico; se ha logrado evidenciar la importancia de implementar otra variable, que es crucial al obtener resultados más significativos, al obtener cambios extrínsecos en el ser humano y es la nutrición, un elemento de gran predominio, al generar cambios representativos, en el porcentaje de grasa corporal, visceral, masa muscular y demás indicadores, que hacen parte de la composición corporal.

\section{REFERENCIAS}

1. ABRALDES VALEIRAS, A. 2016. Principios Fundamentales del Acondicionamiento Físico. (Murcia, España).

2. AZUERO AZUERO, A. 2019. Significatividad del marco metodológico en el desarrollo de proyectos de Investigación. Revista Arbitrada Interdisciplinaria koinonia. 4(8):110-127.

3. BARBADO, C.; BARRANCO, D. 2007. Manual de ciclo indoor avanzado. Paidotribo (Barcelona). $198 p$.
4. CHAVARRIAS, M.; CARLOS-VIVAS, J.; BARRANTESMARTÍN, B.; PÉREZ-GÓMEZ, J. 2019. Effects of 8-week of fitness classes on blood pressure, body composition, and physical fitness. J Sports Med Phys Fitness. 59(12):2066-2074. https:// doi.org/10.23736/S0022-4707.19.09886-4

5. KAYA, F.; NAR, D.; SAID ERZEYBEK, M. 2018. Effect of spinning cycling training on body composition in women. Journal of Education and Training Studies. 6(4):154-160. https:// doi.org/10.11114/jets.v6i4.3028

6. ORGANIZACIÓN MUNDIALDELASALUD-OMS. 2018. Enfermedades no transmisibles. Organización Mundial de Salud. Disponible desde Internet en: https://www.who.int/es/news-room/factsheets/detail/noncommunicable-diseases (con acceso el 30/10/2020).

7. OTZEN, T.; MANTEROLA, C. 2017. Técnicas de Muestreo sobre una Población a Estudio. Int. J. Morphol. 35(1):227-232. http://dx.doi. org/10.4067/S0717-95022017000100037

8. RANDELL, R.K.; ROLLO, I.; ROBERTS, T.J.; DALRYMPLE, K.J.; JEUKENDRUP, A.E.; CARTER, J.M. 2017. Maximal Fat Oxidation Rates in an Athletic Population. Medicine \& Science in Sports \& Exercise. 49(1):133-140. http:// dx.doi.org/10.1249/MSS.0000000000001084

9. VALLE, V.S.; MELLO, D.B.; FORTES, M. DE S.; DANTAS, E.H.; MATTOS, M.A. 2010. Effect of diet and Indoor Cycling on body composición and serum lipid. Arq Bras Cardiol. 95(2):173-178. https://doi.org/10.1590/s006 6-782X2010005000080

10. VILARINHO, R.; GARCEZ DE SOUZA, W.; RODRIGUES, T.C.; AHLIN, J.V.; GUEDES JUNIOR, D.P.; MADUREIRA BARBOSA, F. 2009. Effect of indoor cycling in body composition, muscular endurance, Flexibility, balance and daily activities in physically active elders. Fitness \& Performance Journal. 8(6):446-451. https://doi.org/10.3900/fpj.8.6.446.e

11. WILMORE, J.; COSTILL, D.L. 2004. Fisiología del esfuerzo y del deporte. 5ta Edición. Editorial Paidotribo. $715 p$. 